\title{
Blaze a trail
}

\section{It's been a good month for astronomical discovery and innovation.}

\author{
Alpha Centauri is the closest star system to \\ the Solar System, little more than four light \\ years away. Two of its three stars, Alpha \\ Centauri A and B, are similar to the Sun \\ and now an Earth-mass planet has been \\ spotted $^{1}$ in orbit around Alpha Centauri \\ B. But there the similarity ends: the planet \\ is far too close to its star to harbour any \\ form of life. Yet its discovery is testament \\ to the remarkable sensitivity of exoplanet \\ searches based on radial velocity - picking \\ up the slight 'wobble' in a star's motion \\ due to the gravitational pull of an orbiting \\ planet - this one having been made \\ at the European Southern Observatory \\ in Chile, which this year celebrates its \\ fiftieth anniversary. \\ Just days earlier, an exoplanet \\ discovery of a rather different nature was
}

announced $^{2}$. Nearly 5,000 light years away is a Neptune-size planet trapped in orbit between four stars - the first system of this kind ever observed. What is also remarkable is how it was discovered, through Planethunters.org. This website, set up only two years ago, hosts data from NASA's Kepler space observatory and encourages members of the public to join the exoplanet search by perusing the data for the signature of a transit, the slight dip in a star's brightness as a planet passes in front of it. Two such volunteers spotted the four-star planet, which was followed up and confirmed by astronomers using the Keck telescope in Hawaii.

Meanwhile, across the globe, the International Scientific Optical Network in Russia reported the discovery of a comet,
C/2012 S1 (ISON), that might, by this time next year, be putting on a stunning display. Thought to have originated from the Oort Cloud, and captured in CCD images from a 0.4-metre reflector, comet ISON should reach perihelion and peak brightness in late November 2013. It could be brighter than the full Moon and rival the Great Comet of 1680, whose parabolic orbit was proved by Isaac Newton to fit his law of universal gravitation.

Ground-based or space-based, intragalactic or extragalactic, professional or volunteer - this is astronomy to be proud of, and enjoy.

1. Dumusque, X. et al. Nature http://dx.doi.org/10.1038/ nature11572 (2012).

2. Schwamb, M. E. et al. Preprint at http://arxiv.org/abs/ 1210.3612 (2012).
}

\section{What's in a name?}

\section{A unique identifier for every researcher will keep the scientific record in order.}

I. Newton, B. Franklin, A. Einstein in the smaller scientific community of the past, a surname and initial sufficed to identify a researcher and track their contribution to the scientific literature. But the global, mobile community of the twenty-first century is another matter: so many scientists and so many shared surnames are blurring the picture of attribution.

For example, in 2011 there were nearly 4,000 publications authored by Y. Wang certainly not the same Y. Wang, so how should any one of the Y. Wangs claim proper credit for their own research? Neither is this only a problem for researchers originating from the Far East: a search of Thomson Reuters' Web of Science throws up more than four thousand entries for A. Wright. Which Wright is the right Wright?

Hence the launch of ORCID - the Open Researcher and Contributor ID (www.orcid.org). Every researcher may create, free of charge, a 16-digit machinereadable number as their identifier in the ORCID registry (which is open and non-proprietary), and then link that unique code with their papers or other research output. The result would be a disambiguation of author names, allowing any one author to keep a clear record of their work and, through choice of privacy settings, make that record available to others as they wish.

Nature Publishing Group, to which Nature Physics belongs, is a launch partner of ORCID - one of seventeen, including universities, publishers and funding bodies.

Readers registered on nature. com can now link an ORCID identifier to their nature. com profile; authors submitting to this journal can also link their ORCID identifier to their submission. As further functionality is developed, more features are planned to improve its integration with nature.com and the manuscript-submission system.

We encourage all of our readers and authors to register with ORCID - the benefits of such a registry are plainly contingent on take-up among scientists, as well as on the efforts of publishers and others to incorporate the codes in the process of publishing. It could become a hugely useful Who's Who of science who really is who. 